\title{
HapPro: a wearable haptic device for proprioceptive feedback
}

\author{
Matteo Rossi ${ }^{1,3}$, Matteo Bianchi ${ }^{1,2}$, Edoardo Battaglia ${ }^{1}$, Manuel G. Catalano ${ }^{3}$, and Antonio Bicchi ${ }^{1,2,3}$
}

\begin{abstract}
Objective: Myoelectric hand prostheses have reached a considerable technological level and gained an increasing attention in assistive robotics. However, their abandonment rate remains high, with unintuitive control and lack of sensory feedback being major causes. Among the different types of sensory information, proprioception, e.g. information on hand aperture, is crucial to successfully perform everyday actions. Despite the many attempts in literature to restore and convey this type of feedback, much remains to be done to close the action-perception loop in prosthetic devices. Methods: With this as motivation, in this work we introduce HapPro, a wearable, non-invasive haptic device that can convey proprioceptive information for a prosthetic hand. The device was used with an under-actuated, simple to control anthropomorphic robotic hand, providing information about hand aperture by mapping it to the position of a wheel that can run on the user's forearm. Tests with 43 able bodied subjects and one amputee subject were conducted in order to quantify the effectiveness of HapPro as a feedback device. Results: HapPro provided a good level of accuracy for item discrimination. Participants also reported the device to be intuitive and effective in conveying proprioceptive cues. Similar results were obtained in the proof-of-concept experiment with an amputee subject. Conclusions: Results show that HapPro is able to convey information on the opening of a prosthetic hand in a non-invasive way. Significance: Using this device for proprioceptive feedback could improve usability of myoelectric prostheses, potentially reducing abandonment and increasing quality of life for their users.
\end{abstract}

Index Terms-upper extremity prosthesis, haptic feedback, proprioception

\section{INTRODUCTION}

The loss of a limb is a personally and socially dramatic event, especially for the upper limb, due to the fine motor tasks carried out by the hand and arm. Prostheses have emerged to help with such a physical loss and partially restore the lost functionality. Body-powered and cosmetic prostheses have been used for centuries and are still commonly prescribed today. More recent prostheses use electrically powered actuators controlled via electromyographic (EMG) signals from the muscles in the residual limb. The aim of these myoelectric prostheses is to provide increasing functionality without sacrificing appearance: however, their main drawback is that they are often difficult to control for the user [1].

There are no clear numbers about the use of upperlimb prostheses, but there is indication that, while cosmetic prostheses are largely used, myoelectric prostheses are the

1. Research Center Enrico Piaggio, University of Pisa, Largo Lucio Lazzarino 1, 56126 Pisa, Italy.

2. DII - Department of Information Engineering, University of Pisa, via G. Caruso, 16, 56122, Pisa, Italy.

3. Soft Robotics for Human Cooperation and Rehabilitation, Istituto Italiano di Tecnologia, Via Morego 30, 16163, Genova, Italy.

(e-mail: matteo.rossi@iit.it)

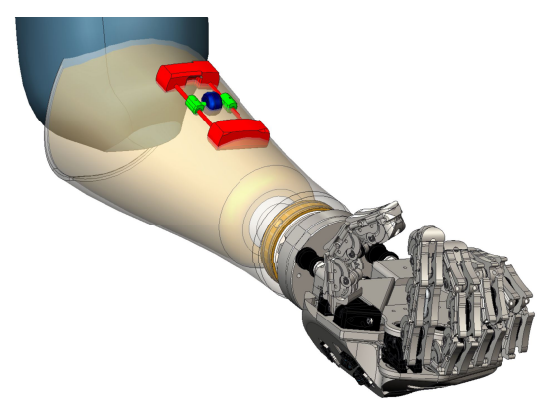

Figure 1: HapPro: concept.

least popular [2]. In fact, despite the advances made in the design of dexterous anthropomorphic hand prostheses, these sophisticated devices tend to disappoint the high expectations of their potential users, who ideally want a prosthesis able to replicate the dexterity, sensitivity, and strength of a human hand, while being at the same time easy to use. This discrepancy between the users' expectations and available products is evident when looking at the rejection rate of upper-limb prostheses; roughly one third of these prescribed prostheses are completely rejected [3]. But which are the factors that lead to the abandonment of these technologically advanced myoelectric prostheses? Pylatiuk et al. [4] performed a study to try to answer this question. Using questionnaires, they found that one of the major factors in myoelectric prostheses abandonment is the lack of sensory feedback. Also, Biddiss et al. [5] state that myoelectric users are not satisfied with the feedback that is available from their prostheses. Indeed, although sensory feedback is of primary importance for prosthesis users [6], to the best of our knowledge, there are no commercial myoelectric prostheses that can provide haptic feedback, and myoelectric users must therefore rely heavily on visual feedback when operating their prosthesis.

Appropriate cutaneous feedback can relieve the users of the burden of having to keep constant visual contact with the prosthesis, and help drive a perceptual shift towards embodiment of the device for these amputees [7]. In order to implement more natural control interfaces, therefore increasing acceptance, the research community is attempting to close the control loop between the patient and the prosthesis by actively pursuing various methods of providing haptic feedback to prosthesis users. Generally, a feedback system registers the variables of interest with appropriate sensors, and translates the acquired information to the user, with invasive or non-invasive methods. Typical non-invasive methods include vibrotactile feedback [8], electrotactile feedback [9], force feedback [10] and skin stretch [11]. By using these modalities, it is possible 
to convey information on the status of a prosthesis to its user. For instance, Ninu et al. [8] used vibrotactile stimulation to provide feedback on the closing velocity and grasping force of a hand prosthesis; Casini et al. [12] designed a wearable device able to deliver grasping force information via mechano-tactile stimulation of the user's arm skin, while Bark et al. [11] built a rotational skin stretch device for proprioceptive feedback.

Although sensory feedback can bring many benefits to amputees, it should be emphasized that the simultaneous display of multiple types of haptic information does not necessarily enhance grip force control. On the contrary, it can degrade it [13]. It is therefore important to identify what information is most important for task accomplishment and the best manner to convey it back to the users. When dealing with non-sighted conditions, proprioception plays a critical role in enabling humans to precisely control their movements [14]; not surprisingly, amputees are demanding prostheses that can be operated without constant visual attention [4], [15].

For these reasons, the use of skin stretch, electrotactile and vibrotactile feedback to convey proprioceptive information has been investigated [9], [11], [16]. As described in the literature, these feedback modalities can indeed be used to convey useful information on the position of the prosthesis to the amputee; however, they all share the inconvenience of requiring to be constantly active to convey information, which can be impractical for use in everyday life. Contrary to the aforementioned methods, a displacement-based display would not require continuous activation to maintain a response from slow-acting mechanoreceptors, and therefore would provide continuous proprioceptive awareness. However, to the best of our knowledge, this kind of stimulation has never been investigated for proprioceptive restitution. Furthermore, evaluation of effectiveness of haptic feedback to convey information on the pose of a prosthetic hand was always done in a virtual reality environment, and never in a test where a prosthetic hand was physically interacting with objects.

In an attempt to fill this gap, we designed a novel wearable haptic device, named HapPro, to convey proprioceptive information of a prosthetic hand through tactile stimulation. In this work we focus on trans-radial amputees, and thus study stimulation on the forearm's skin with the aim of integrating the device inside the prosthetic socket. The device is constructed of a structure of plastic and aluminum and a system of pulleys, actuated by a DC motor, that controls the position of the end-effector, a plastic wheel. Our approach maps the level of hand aperture to the position of the wheel; the elicited sensation is therefore indicative of both position and velocity of hand closure.

The HapPro was designed to work with, and be connected to, the SoftHand Pro (SHP), an anthropomorphic robotic hand with 19 degrees of freedom that uses just one motor to activate its adaptive synergy [17]. The idea behind the HapPro is quite intuitive: the end-effector of the HapPro moves as if it was connected to an imaginary tendon driving the SoftHand Pro. If the HapPro is worn on the anterior side of the forearm, when the SoftHand Pro closes, the wheel moves proximally, and when the hand opens, the wheel is "pulled" distally. This type of feedback is intrinsically synergy-based [18], in the sense that the information conveyed to the user is proportional to the motions of the SoftHand Pro, which, by construction, follow a synergistic path [19].

Experiments were performed to evaluate the device and its effectiveness in conveying proprioceptive feedback. In a first experiment we investigated the just noticeable difference for the stimuli produced by the HapPro in different locations of the forearm. Then, we tested the ability of the subjects to discriminate between objects of different sizes that were grasped with the SoftHand Pro, while relying only on the feedback conveyed by the HapPro. Overall, 43 able-bodied subjects and one transradial amputee participated in the experiments. To the best of our knowledge this is the first time that a feedback device is used to convey proprioceptive information of a hand prosthesis to an amputee.

\section{BACKGROUND}

Proprioception, from the Latin "proprius" (one's own) and "căpĭo" (to take, to obtain), is the ability to perceive the position and movements of the body. Without looking at it, a person is able to sense the posture of the hand as well as its movements; this apparently simple task is made possible by the information provided by a combination of receptors, which include cutaneous mechanoreceptors, Golgi tendon organs, joint receptors and muscle spindles [20], [21]. There is evidence that being able to know the status of a prosthetic hand without visual contact is a highly desirable feature for users [15], [4]. The inclusion of proprioceptive feedback would address this request, and could help reduce the rejection rate for upper limb prostheses.

Previous work has shown the potential of artificial proprioception in prosthetics. Blank et al. [14] performed a study with able-bodied subjects in a virtual reality environment, showing that, in absence of visual feedback, proprioceptive motion feedback always improves targeting accuracy, and that it can also be beneficial under sighted conditions. More recently, Pistohl et al. tested the ability of controlling a cursor via EMG signals on a group of able-bodied subjects, with and without proprioceptive feedback [22]. The EMG signals were measured from muscles of the left hand, while proprioceptive feedback was provided by moving the right arm with a robotic manipulandum. Results showed that the overall performance was unaffected by the additional feedback when visual feedback was available, while the artificial proprioceptive feedback supplied to the contralateral arm significantly improved myoelectric control under unsighted conditions.

Providing feedback to a prosthesis user is typically a twostep process: 1) the variable of interest must be sensed on the prosthesis side, and 2), the information has to be relayed to the user. Concerning proprioceptive feedback, the first step is relatively straightforward, since most of the prosthetic hands currently available are equipped with built-in position sensors. For the second step, a non-invasive option to convey proprioception is offered by sensory substitution through haptic displays. In 1970, Mann and Reimers showed that the use of a vibrotactile display of elbow's angle improved the ability of positioning an EMG-controlled prosthesis [23]. In more recent studies, information on the pose of an EMG controlled virtual hand was conveyed by tactors positioned on the forearm [24] or on the waist [18] of the participants; the vibrotactile feedback was found to improve control accuracy in absence of visual feedback. Vibrotactile feedback can be 
relayed by using compact and inexpensive actuators. For this reason, vibrotactile actuators have been extensively studied as a feedback method and it is often used as a baseline standard for comparison with other feedback methods [25]. However, not all of the studies conducted on vibrotactile feedback for proprioception have shown promising results [26]. Other feedback modalities that have been used to convey proprioceptive information include electrocutaneous stimulation and skin stretch. Electrotactile feedback is based on the application of small currents to the skin of the prosthetic user. Although the low cost and compactness of the electrodes would make electrocutaneous stimulation ideally very suitable for a feedback application, it has been shown to produce a range of unpleasant sensations [27], and to perform worse when compared with vibrotactile stimulation [9].

Skin stretch was also investigated: Akhtar et al. used passive linear skin stretch devices to provide posture feedback of a 3D printed robotic hand [16], while Wheeler et al. [28] provided a sense of position and motion of a virtual elbow by inducing rotational skin stretch on the participants' skin. When compared with vibrotactile feedback, skin stretch showed no significant difference in performance [16] in one study, and provided superior results in another [29]. As pointed out by Bark et al. [29], an advantage of active skin stretch over vibrotactile feedback is its intrinsic ability to simultaneously display position and motion and to convey positive or negative direction without the use of multiple stimulators. However, compared with other displays, these devices are based on force or torque control, which can cause stability problems. Another drawback of skin stretch methods is the temporary nature of their connection with the skin: in order to transmit motions, contact pads need to be attached to the skin with adhesive; the effectiveness and consistency in reapplication of these connections have yet to be proved [25].

In this paper we present a wearable haptic device that conveys proprioceptive information through a moving contact on the skin's surface. The design is conceptually similar to one of the cases described by Provancher et al. [30], in which wheel-based system was used to stimulate the fingertips of a subject, but this approach has never been used as a feedback modality for conveying proprioception. The aim of our study is to analyze using such a displacement based display for proprioceptive feedback. More specifically we evaluate usage with the SoftHand Pro [31], the prosthetic version of the Pisa/IIT SoftHand (SH) [17]. This under-actuated and adaptable artificial hand is designed to move according to the most common pattern of grasping in humans [19], or first postural synergy, using a single motor and tendons routing to move the fingers in a coordinated fashion. When used in combination with the SoftHand Pro, the HapPro provides information on the overall level of hand closing along the first synergy.

\section{DEVICES}

\section{A. HapPro Design}

The HapPro is a wearable cart-like structure, with a pulleybased system that transforms rotational motion from a DC motor into linear motion. Fig. 2(a) shows an exploded view of the system. The DC motor (Pololu 298:1 Micro Metal
Gearmotor) is placed on a support, and has on its shaft a pulley housing a magnet for an absolute magnetic encoder (12 bit magnetic encoder AS5045 by Austria Microsystems). The encoder measures the position of the motor, and therefore can be used to compute the position of the cart. In this paper we refer to the encoder measure as $q_{\text {Hmeas }}$. A second support hosts a second pulley, and is placed on the other side of the device.

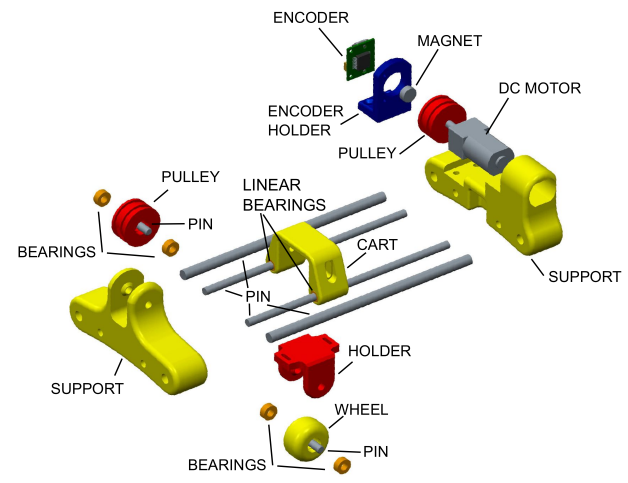

(a)

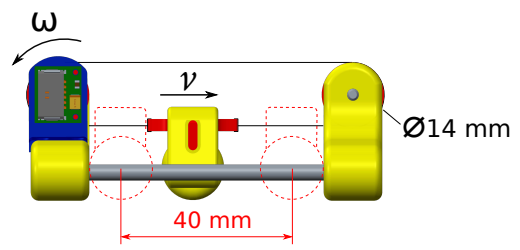

(b)

Figure 2: HapPro design. (a) shows the exploded view of the HapPro highlighting all of its mechanical components. (b) shows the side view of the HapPro and the movement mechanism: an angular velocity $\omega$, imposed by the motor, generates a linear movement of the cart with velocity $v$ proportional to $\omega$ and the pulley radius $R: v=\omega R$. In this configuration, as shown in figure, the maximum linear displacement of the cart is $40 \mathrm{~mm}$.

The two supports are joined by four bars, of which two are larger (4 mm diameter) and hold the device on the arm, while the remaining two are smaller ( $2 \mathrm{~mm}$ diameter) and are used as guides for a cart. The cart itself can move over the smaller pins with low friction, thanks to two linear bearings, and holds a wheel that can roll on the arm during the cart movement. The wheel holder is designed so that two wires can be wrapped around it. These wires are tightly wrapped around the supports pulleys, so that the motor rotation moves the cart (Fig. 2(b)): in particular, the linear movement of the cart can be simply obtained from the encoder readings and the pulley radius.

Fig. 3 shows an image of the device on the subject arm. As can be seen the device is fairly unobtrusive, and has a total weight of $75 \mathrm{~g}$. A custom made electronic board (PSoC-based electronic board with RS485 communication protocol) controls motor positions based on the readings from the encoder. More details on the system control methods are provided in the following sections. All the structural parts of the HapPro except for the pins are in ABSplus - Stratasys, 


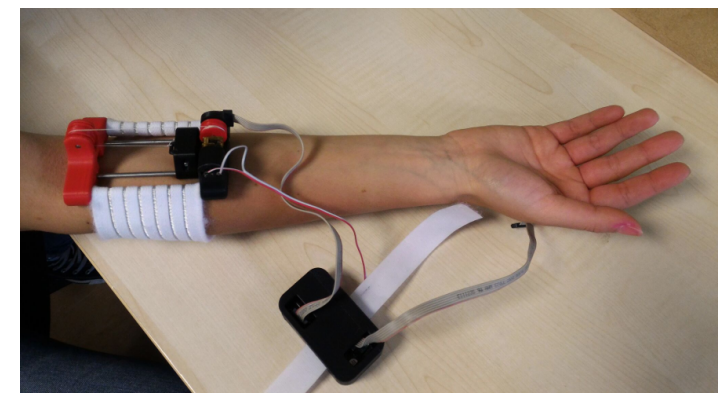

Figure 3: HapPro: physical prototype.

rapid prototyping material.

\section{B. The SoftHand Pro}

The SoftHand Pro [31] is the myoelectric version of the Pisa/IIT SoftHand. The Pisa/IIT SoftHand design [17] takes inspiration from research in neuroscience. It is well known that humans control their hands not merely by acting on each of its numerous degrees of freedom, and by coordinating and coactivating them in organized motions called synergies ([19], [32]). Soft synergies, introduced in [33], are a more recent concept, where the synergy serves as a reference position for a virtual hand, and the interaction forces between the hand and a grasped object depend on the stiffness matrix connecting the virtual and real hand position.

The Pisa/IIT SoftHand combines compliance and synergy inspiration into an artificial hand with 19 DoFs, 4 on each of 4 fingers, and 3 on the thumb. The fingers are capable of flexion/extension as well as ab/adduction. For ab/adduction of the fingers and at the equivalent of the carpometacarpal joint of the thumb (responsible for rotating the thumb from lateral pinch to $\mathrm{C}$ grasp, for example), traditional revolute joints were employed. The rest of the joints incorporate rolling contact joints with elastic ligaments, which ensure physiologically correct motions when actuated, and easily disengage on impact to allow safe interaction with humans. The elastic ligaments also allow deformation while ensuring the hand returns to its original configuration. A single tendon runs though all joints to simultaneously flex and abduct the fingers upon actuation.

The hand is actuated by a single DC motor which moves the fingers on the path of the first synergy as described in [19]. However due its compliant yet robust design, it can mold around a large variety of objects. The motor employed in the current release is a 15 Watt Maxon DCX 22S with a GPX22 (86:1) gearhead and a 12 bit magnetic encoder, resolution of $0.0875^{\circ}$ (Austrian Microsystems), which can be used to measure the hand aperture. In this paper we refer to the value obtained from this encoder with $q_{\text {Smeas }}$. With this setup the hand has a maximum force of $130 \mathrm{~N}$ perpendicular to the palm. The CAD model of the Pisa/IIT SoftHand, as well as the design of the electronic board used in this work are open source and available at the Natural Machine Motion Initiative Website (http://www.naturalmachinemotioninitiative.net/) [34].

Sharing the same mechanical design with the SoftHand, the SoftHand Pro is characterized by the same adaptability, resilience to high forces, robustness and reliability described above. In addition, the SoftHand Pro features a myoelectric control interface that allows users to control the hand using commercial surface electromyography (EMG) sensors (Otto Bock, Germany). These sensors detect the electrical activity from the patient's arm muscles, and it is possible to control the hand by appropriate muscle contractions.

\section{Control Strategy}

The block diagram in Fig. 4 describes the closed-loop control scheme of the SoftHand Pro with the human in the loop. The electromyographic activity from the FDS and the EDC muscles is measured from the skin of the user's forearm using two Ottobock 13E200 sensors (Otto Bock HealthCare $\mathrm{GmbH}$, http://www.ottobock.com/), which directly provide an amplified, bandpass-filtered, and rectified version of the raw EMG signals. The EMG signals are acquired and their difference is computed. The desired velocity $\dot{q}_{S r e f}$ for the SoftHand Pro is then set proportional to that difference so that when the FDS generates a higher EMG signal than the EDC, the hand is closing, when the EDC generates a higher EMG signal than the FDS, the hand is opening and when the difference between the two EMG signals is below a threshold, the desired velocity is set to zero. The measured position of the motor of the SoftHand Pro $q_{\text {Smeas }}$ is then mapped to the reference position for the HapPro $q_{\mathrm{Href}}$, i.e. every movement of the hand produces a movement of the HapPro end-effector, generating a tactile stimulation of the forearm skin.

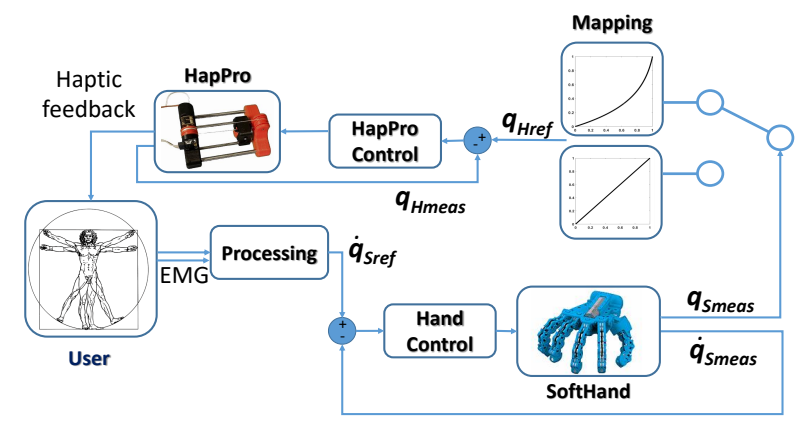

Figure 4: Block diagram of the SoftHand Pro, controlled by the user with EMG sensors, with haptic feedback in the loop.

The tactile stimulation performed by the HapPro directly depends on the value of the SoftHand Pro encoder $q_{\text {Smeas }}$; this information can be used to understand whether the hand is grasping an object, and, if so, it can also give insight on the dimension of the object that is being grasped. However, given the dimension of the null-space of the SoftHand Pro, it can happen that the same object will produce different $q_{\text {Smeas }}$ if grasped multiple times, and that objects of different size will correspond to the same value of $q_{\text {Smeas }}$.

To choose a suitable set of objects for the size discrimination test, we selected eight wooden spheres of different diameters (Fig. 5) and recorded the $q_{\text {Smeas }}$ values during the grasps. In particular, each sphere was positioned in the palm of the SoftHand Pro and $\dot{q}_{\text {Sref }}>0$ was set so that the SoftHand Pro closed completely around the sphere. When the grasping phase was complete and $\dot{q}_{\text {Smeas }}$ was zero, we recorded the value of $q_{\text {Smeas }}$. This procedure was repeated ten times for each sphere. We also recorded $q_{\text {Smeas }}$ when the SoftHand Pro was 


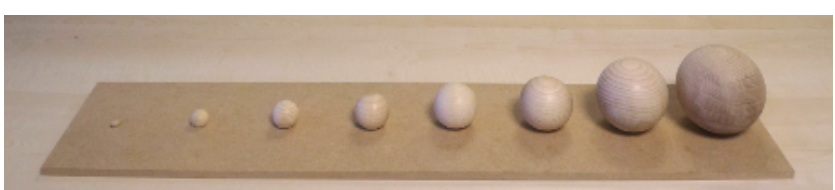

Figure 5: Wooden spheres of different sizes. From left to right the diameters are: $10 \mathrm{~mm}, 20 \mathrm{~mm}, 30 \mathrm{~mm}, 40 \mathrm{~mm}, 50 \mathrm{~mm}$, $60 \mathrm{~mm}, 80 \mathrm{~mm}, 95 \mathrm{~mm}$

completely open and completely closed in absence of objects. The box plot of the encoder values obtained during these grasp repetitions is presented in Fig. 6. In each box, the central mark represents median, while the edges of the box are the 25 th and 75th percentiles. Minimum and maximum values for each subset of grasps are represented by the horizontal indents.

In the box plot there is overlap between some of the different sphere sizes. If a subject's ability to discriminate the size of the grasped objects with the HapPro using objects that, when grasped, can produce the same value of $q_{\text {Smeas }}$, the results would not be a reliable measure of the HapPro usefulness. In order to obtain a meaningful set that could be used for the tests, we therefore extracted the largest subset of cases with non-overlapping boxes: $\{$ closed hand, $\oslash 10 \mathrm{~mm}, \oslash 30 \mathrm{~mm}$ $\oslash 50 \mathrm{~mm}, \oslash 80 \mathrm{~mm}$, open hand $\}$.

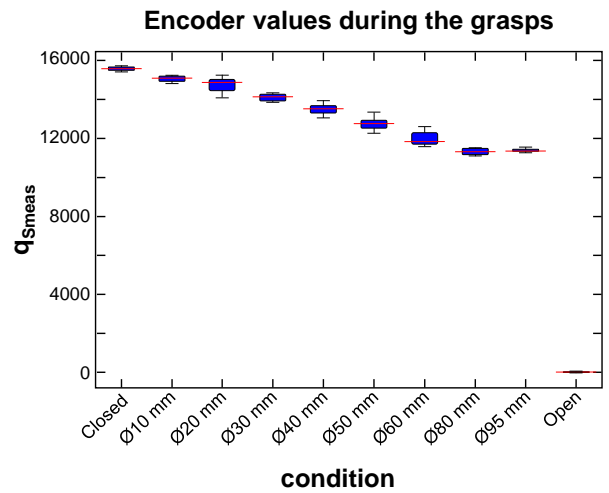

Figure 6: Box plot of the values of $q_{\text {Smeas }}$, recorded during the grasp of different spheres.

The value of $q_{\text {Smeas }}$ is sent from the SoftHand Pro control board to the HapPro control board via RS485 at a frequency of $1 \mathrm{kHz} ; q_{\text {Smeas }}$ is then mapped to a reference position $q_{H r e f}$ for the HapPro. Mapping $q_{\text {Smeas }}$ to $q_{H r e f}$ means using a function to associate each value of $q_{\text {Smeas }}$ to a value of $q_{H r e f}$. The simplest mapping to use is perhaps the linear mapping. The linear mapping consists of a simple scaling: the constraints imposed are that $q_{\text {Smeas }}=0$ corresponds to $q_{\text {Href }}=0$ and $q_{\text {Smeas }}=q_{\text {Smax }}$ to $q_{\text {Href }}=q_{\text {Hmax }}$, being $q_{\text {Smax }}$ the maximum value of the SoftHand Pro encoder and $q_{H \max }$ the maximum value of the HapPro encoder. Therefore, $q_{\text {Href }}$ is computed as:

$$
q_{\text {Href }}=\left(\frac{q_{\text {Hmax }}}{q_{\text {Smax }}}\right) q_{\text {Smeas }}
$$

The box plot in Fig. 6 shows that the values of $q_{\text {Smeas }}$ relative to the grasp of the spheres are concentrated in the upper third of the range. This means that if the goal is to discriminate the size of these spheres based on the tactile stimulation $q_{\text {Hmeas }}$, a non-linear mapping could be a better choice, since it can be used to obtain a "magnifying" effect over the mapped domain. For this work, we therefore chose to test also a logarithmic mapping, with the same constraints used for the linear mapping: $q_{\text {Smeas }}=0$ corresponds to $q_{\text {Href }}=0$ and $q_{\text {Smeas }}=q_{\text {Smax }}$ to $q_{\text {Href }}=q_{\text {Hmax }}$. Additionally, for the reason previously explained, we imposed that $q_{\text {Smeas }}=\frac{2}{3} q_{\text {Smax }}$ is mapped to $q_{\text {Href }}=\frac{1}{3} q_{\text {Hmax }}$. These constraints are imposed by choosing the appropriate values for the constants $\alpha$ and $\beta$ : $\alpha=0.9510$ and $\beta=-0.3317$.

$$
q_{\text {Href }}=\beta \log \left(1-\alpha \frac{q_{\text {Smeas }}}{q_{\text {Smax }}}\right) q_{\text {Hmax }}
$$

Graphical representations of the two mappings are shown in Fig. 7.

\section{EXPERIMENTAL PROTOCOL}

A total of 43 healthy subjects volunteered for the studies. 23 were male, mean age $27 \pm 3$ years. The participants were right-hand dominant and did not suffer from any cognitive impairment, that could interfere with their ability to follow the instructions of the study, nor any pathology that could affect tactile sensibility or muscular activity of the forearm. The methods and procedures described in this paper were approved by the Ethical Committee of University of Pisa and the volunteers signed an informed consent before participating. Subjects were allowed to take self-timed breaks and were encouraged to rest if they were showing slight symptoms of fatigue.

Twelve subjects (six female) participated in the first experiment, in which the method of constant stimuli was used to find the just noticeable difference (JND) for the stimuli produced by the HapPro. Fifteen subjects participated to the second experiment, which tested their ability to discriminate between spheres of different sizes, using the SoftHand together with the HapPro. Finally, the remaining sixteen subjects took part in the last experiment in which they completed the same size discrimination task, this time while moving the prosthetic hand autonomously to place it over cylinders and grasp them. All subjects tolerated the protocol well.

\section{A. Just Noticeable Difference}

In this experiment, we used the method of constant stimuli to find the just noticeable difference (JND) as defined in [35], i.e, the minimum amount of distance traveled by the wheel that can elicit a different perception in users with respect to a reference stimulus. Subjects were seated comfortably with the right forearm leaning on a desk, with the elbow at approximately 90 degrees. The HapPro was fastened around the proximal third of the right forearm with orthopedic elastic Velcro bands. The experiment consisted of two sub-parts for each subject: in sub-part A subjects had the palm of the right hand facing up and the HapPro on the anterior side of the forearm, in sub-part B subjects had the palm facing down and the HapPro on the posterior side. The two sub-parts were performed similarly in every other aspect, as detailed in the following paragraphs. Half of the subjects started with subpart $\mathrm{A}$ and then performed sub-part $\mathrm{B}$, while the other half followed the opposite order. 


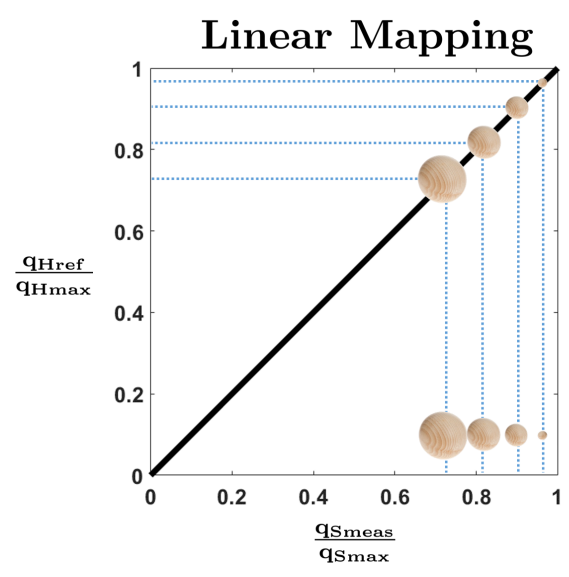

(a)

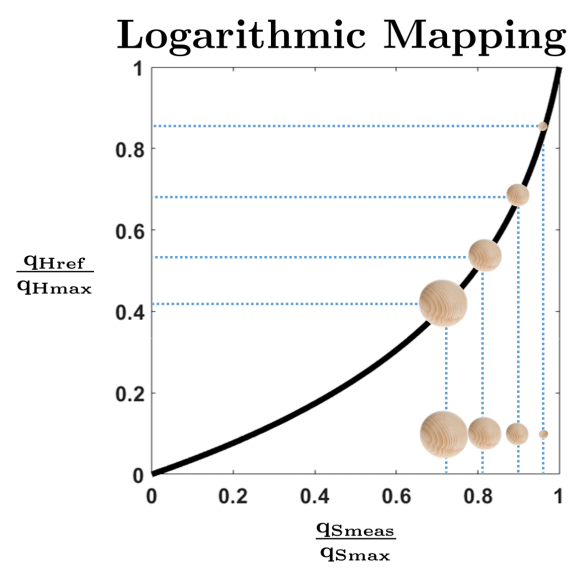

(b)

Figure 7: Two different mappings between the measured position of the SoftHand Pro motor $\left(q_{S m e a s}\right)$ and the reference position for the HapPro $\left(q_{\mathrm{Href}}\right)$. (a) represents the linear mapping between the normalized values of $q_{\text {Smeas }}$ and the normalized values of $q_{H r e f}$, while (b)is a graphical representation of the logarithmic mapping between the normalized values of $q_{\text {Smeas }}$ and the normalized values of $q_{H r e f}$.

Subjects received paired stimuli, each stimulus consisting of a displacement of the wheel followed by its return to the starting position, and were asked to indicate which stimulus in the pair corresponded to the longer displacement. Each pair consisted of a standard stimulus (St) of $20 \mathrm{~mm}$ and a comparison stimulus (Co), presented in random order. We used five equally spaced comparison stimuli ranging from 10 to $30 \mathrm{~mm}$. In a preliminary study, the minimum and maximum displacements used were chosen such that they were almost always judged as less than or greater than the standard stimulus St. The maximum value chosen for the displacement in this experiment is a consequence of the range of motion of the HapPro, which is limited since the envisioned usage of this device is as an integrated component inside a prosthetic socket. A single trial consisted of: the first stimulus, an interstimuli interval $(1 \mathrm{~s})$, and the second stimulus, followed by the subject's response. The subjects had been instructed to respond by saying either "1" or "2" as quickly as possible after the presentation of the paired stimuli to indicate whether the stimulus with longer displacement was respectively the first or the second of the pair. No fixed time within which the subject was forced to respond was imposed. To prevent the subjects from using timing cues, the speed of motion was varied so that each stimulation was completed in a fixed time (1 s), similar to the approach adopted in [36]. References [37], [36] provide more examples of application of JND.

Each sub-part lasted approximately 10 minutes, and consisted of 100 paired stimuli presented randomly (20 pairwise discrimination for 5 stimulus levels). Randomization was done independently for each stimuli sequence. Each pair was presented at least two seconds after the previous one and the two sub-parts were separated by a five-minute break. During both sub-parts, the subjects wore headphones playing white noise to prevent auditory cues. In addition, a screen was used to occlude their right arm and the HapPro from the visual field.

\section{B. Sphere-size discrimination task}

The second experimental setup was designed to evaluate the effectiveness of the feedback provided by the HapPro when using the SoftHand for a sphere-size discrimination task. Subjects were seated comfortably in front of a table. The HapPro was fastened around the proximal third of the right forearm with orthopedic elastic Velcro bands. The SoftHand was secured to the right hand and forearm using Velcro bands and the EMG sensors were positioned on the Flexor Digitorum Superficialis (FDS) and on the Extensor Digitorum Communis (EDC) and held in place by an orthopedic elastic band (Fig. 8).

The fifteen subjects were randomly divided into three groups of equal size; for the members of Group I, a linear mapping between the HapPro and the SoftHand was chosen, for Group II, a logarithmic mapping was used and for the members of Group III, the control group, the HapPro was off.

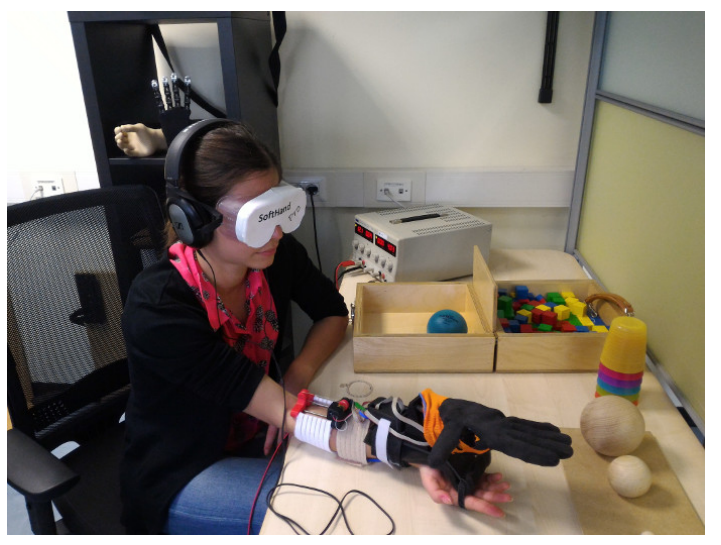

Figure 8: Experimental setup used for the sphere-size discrimination task. Headphones and modified goggles were used to avoid the use of possible auditory and visual cues during the test.

The experiment lasted roughly one hour and consisted of two phases. During the training phase, which lasted approximately 35 minutes, the subjects learned how to use the SoftHand to grasp and manipulate objects of various sizes. Tasks included building a pyramid with plastic glasses, stacking wooden cubes, manipulating wooden spheres and performing 
bi-manual tasks with the help of the left hand. Neither vision nor hearing were obstructed during this phase and, at the end of the training, all of the subjects were able to open and close the hand in a gradual way and grasp objects of various size and compliance without dropping or squeezing them.

During the test phase, which lasted on average around 20 minutes, the subjects wore a headset and their right arm was occluded from view by modified goggles. Pink noise was played through the headphones in order to cover possible auditory cues produced by the actuators of the SoftHand and the HapPro. Subjects were presented fifteen different pairs of spheres and asked to tell whether the second sphere was bigger, smaller or of equal size with respect to the first sphere. To avoid artifacts in the results due to time error, each pair of spheres was presented two times, one with the first element of the pair being presented as first and another one with the first element of the pair presented as second in temporal order, for a total of thirty trials in random order. During each trial, the subjects voluntarily commanded a total closure of the SoftHand to grasp each sphere, and relied on the feedback from the HapPro to infer the size of the sphere. The SoftHand would close less when grasping a sphere of larger size, which would cause a different feedback to be provided based on the encoder reading.

At the end of the test phase, the subjects took off the goggles and the headphones and had the possibility to use the SoftHand for an additional five minutes, so that they could compare the two conditions in a subjective evaluation. For the members of Group I and II the HapPro was turned-off and the subject used the SoftHand without haptic feedback; the members of Group III used the SoftHand with haptic feedback using the HapPro with logarithmic mapping. Finally, at the end of the session, the subjects took off the SoftHand and the HapPro and completed a short survey.

\section{Subjective Evaluation}

At the end of the sphere-size discrimination experiment, the subjects from Group I and II answered a questionnaire of 18 questions using bipolar Likert-type seven-point scales. They were first asked to briefly use the artificial hand without the integration of the HapPro system for 5 minutes. The questionnaire considered the comfort and usability of the proposed experimental setup (four questions), the perceived performance (eight questions), the experimental conditions (four questions) and the level of engagement of the subjects (two questions). To each question, the subject answered by choosing a value between 1 and 7 , with 1 corresponding to Strongly disagree, 7 to Strongly agree, 5 to Undecided and the other values having intermediate valence.

\section{Cylinder-size discrimination task}

While the sphere-size discrimination task provided valuable data to assess the ability of the HapPro to convey an appropriate level of proprioceptive information of the SoftHand Pro, it is interesting to evaluate whether the feedback provided by the HapPro can be effective when the prosthetic hand is being moved autonomously. For this reason, a third experiment was performed by 16 subjects (Fig. 9). The hand setup was identical to the one described in subsection V-B, but the objects to discriminate were cylinders that had to be actively found by the subject (who had their vision blocked using the same goggles). In this case, subjects were divided only in two groups: for 8 subjects the HapPro was not active (condition HapPro OFF), while for the other 8 subjects the HapPro was used with the logarithmic mapping (condition HapPro ON). After the same training phase, the subject wore a headset that played pink noise and their visual field was occluded by modified goggles.

Instead of sitting, the subject was standing in front of a desk with a $1 \mathrm{~m}$ long prismatic structure with four cylinders along the four long edges. The cylinders had the same diameters of the spheres used for the previously described experiment: $\{\oslash 10 \mathrm{~mm}, \oslash 30 \mathrm{~mm} \oslash 50 \mathrm{~mm}, \oslash 80 \mathrm{~mm}\}$.

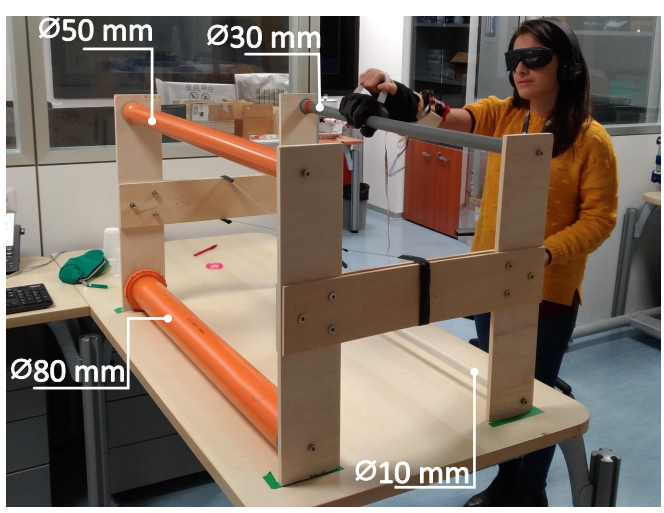

Figure 9: Experimental setup used for the cylinder-size discrimination task. The modified goggles do not allow the subject to use visual feedback to complete the task, while the pink noise played by the headset covers the noise made by the motor of the SoftHand Pro.

Subjects were presented ten pairs of cylinders and asked to tell whether the second cylinder was bigger, smaller or of equal size with respect to the first cylinder. To avoid artifacts in the results due to time error, each pair of cylinders was presented four times, two with the first element of the pair being presented as first and two with the first element of the pair presented as second in temporal order, for a total of forty trials in random order. Each cylinder was always presented at the same distance from the subject. Every time a cylinder was presented, the subjects had to lift the SoftHand above the structure and then lower it until it barely touched the cylinder. The subject then closed the SoftHand Pro, re-opened it and moved it back to the body while waiting for the next cylinder.

\section{STATISTICAL ANALYSIS}

\section{A. Just Noticeable Difference}

We modeled the responses of each volunteer using a psychometric function. In each experimental condition, i.e. body location for stimulus delivery (anterior surface of the forearm - hereinafter referred to as condition ant -, and posterior surface of the forearm - hereinafter referred to as condition post), the psychometric function related the physical spatial displacement of the device sliding over the user's skin with the perceived displacement. Each psychometric function had the form

$$
\Phi^{-1}\left[P\left(Y_{j}=1\right)\right] \sim \beta_{0}+\beta_{1} x_{j}
$$


where $\left[P\left(Y_{j}=1\right)\right]$ is the probability that, in trial $j$, the participant reported a spatial displacement in the comparison larger than the reference stimulus, $\Phi^{-1}[\cdot]$ is the probit transformation of the response probability (i.e., the inverse function of the cumulative normal distribution), and $x_{j}$ is the value of the wheel displacement in the comparison stimulus.

We used a Generalized Linear Mixed Model (GLMM) [38] to extend the psychometric function to the whole pool of participants. This model enabled cluster data analysis [37], [36], in our case a cluster is the collection of repeated responses in several participants. GLMM is a hierarchical model that provides both predictions on the experimental effects, which are assumed to be systematic across participants, and an estimate of the variability between participants. For each experimental condition, we estimated the Just Noticeable Difference (JND), i.e. the amount of stimulus change to detect the just noticeable difference, the Point of Subjective Equality (PSE), i.e. the stimulus value yielding a response probability of 0.5 , with the related $95 \%$ confidence intervals (CIs), using the bootstrap method [38].

\section{B. Size discrimination experiments}

The non-parametric Kruskal Wallis test was used to compare the overall accuracy for multiple groups in the sphere-size discrimination task, while Mann-Whitney $U$ test was used to evaluate the pairwise performance inside the same group and in the cylinder-size discrimination experiments. Post hoc analyses were carried out using Tukey-Kramer correction for multiple comparisons. Mann-Whitney $U$ tests were used to compare Likert scale scores from the subjective evaluation. The significance level $\alpha$ was evaluated at $\alpha=0.05$.

\section{RESULTS}

\section{A. Just Noticeable Difference}

In this experiment, we modeled the responses of each volunteer using a psychometric function. Results of the bootstrap method revealed that the $95 \%$ CI of the difference between PSEs, i.e. $P S E_{\text {post }}-P S E_{\text {ant }}$, includes zero [-0.80; 0.64]. This result implies that this difference is not significant, as expected, and serves as an internal controller of the correctness of our experimental procedure. On the other hand, the $95 \% \mathrm{CI}$ of the difference between JNDs, i.e. $J N D_{\text {post }}-J N D_{\text {ant }}$, does not include zero [-0.98; -0.07$]$. This means that the difference is significant, i.e. the skin on the anterior side is more sensitive $(\mathrm{JND}=3.10 \mathrm{~mm}$ for the ant condition, JND $=3.60 \mathrm{~mm}$ for the post condition) than the skin on the posterior side of the forearm. The GLMM fit is reported in Fig. 10. Leveraging upon these results, we chose the anterior side of the user's forearm for our device, and used it for the other experiments reported in this manuscript.

\section{B. Sphere-size discrimination task}

As noted above, fifteen subjects participated in size discrimination experiments. The spheres used during the test were selected in Section IV, i.e. 10, 30, 50 and $80 \mathrm{~mm}$ as well as the closed and open hand condition. Fig. 11 shows the discrimination accuracy for each subject. Orange bars represent the accuracy obtained by subjects from Group I, blue

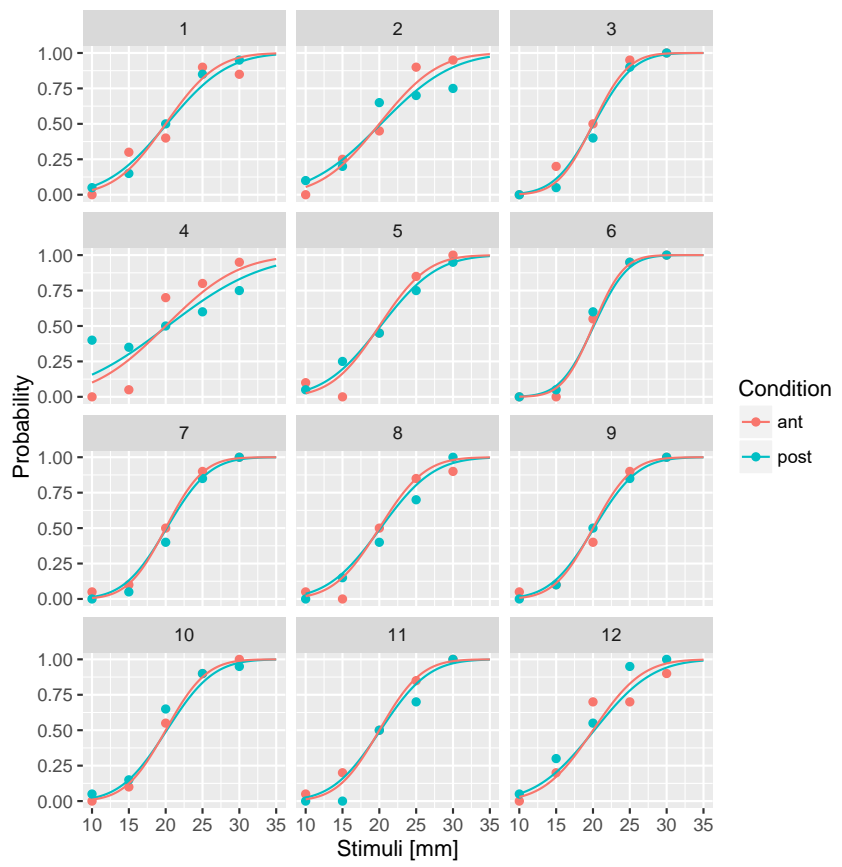

Figure 10: Raw data and GLMM predictions for the anterior (ant) and posterior surface (post) conditions, for all the subjects. Each box represents raw data and model predictions for each single participant (labeled as 1 - 12).

bars represent Group II and white bars represent Group III. The best performance was obtained by the subjects who used the HapPro with the logarithmic mapping, with an average accuracy of $76.7 \%$. Members of Group I obtained an average accuracy of $54.7 \%$, which is still significantly above the chance level. As expected, the accuracy of the members of Group III did not significantly differ from chance $(33.3 \%)$.

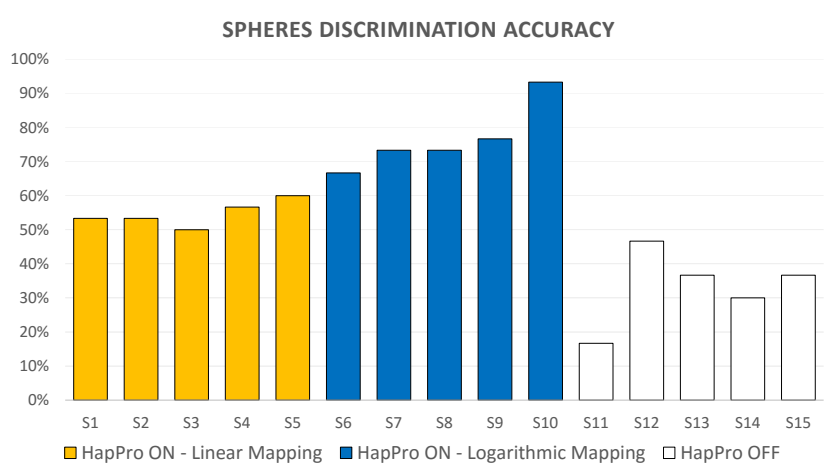

Figure 11: Discrimination accuracy obtained by the members of the three groups during the sphere-size discrimination task.

We performed a statistical analysis based on non-parametric tests. Kruskal Wallis test was used to compare the overall accuracy performance between the three groups, while MannWhitney $\mathrm{U}$ test was used to evaluate the pairwise performance inside the same group. Tukey-Kramer post-hoc correction was adopted. As expected, there is a significant difference in terms of performance between the three groups $(p=0.001)$. A post-hoc analysis with Tukey-Kramer correction reveals that 
a statistically significant difference can be observed between Group II and Group III $(p=0.001)$. This result confirms that logarithmic mapping facilitates proprioceptive clues about artificial hand aperture. Fig. 12 presents the average pairwise accuracy for each group. Considering the comparisons between spheres with very dissimilar dimensions (highlighted with a thick border in the figure), accuracy for Group I and Group II, respectively $76.7 \%$ and $93.0 \%$, was significantly higher than the accuracy obtained for the comparisons between spheres with similar dimensions, respectively $40.0 \%(p=0.009)$ and $61.1 \%(p=0.006)$, as results from a post-hoc analysis with Tukey-Kramer correction. For Group III this difference was not significant: $41.7 \%$ vs. $27.8 \%(p>0.05)$.

\section{Subjective Evaluation}

At the end of the sphere-size discrimination experiment, the subjects from Group I and II answered a questionnaire of 18 questions using bipolar Likert-type seven-point scales. To each question, the subject had answer by choosing a value between 1 (strongly disagree) and 7 (strongly agree). The evaluation of each question is reported in Table I.

Results show using HapPro system was intuitive (Q1) and enables the system to convey important proprioceptive cues for hand opening $(\mathrm{Q} 9, \mathrm{Q} 10)$, which ultimately resulted in a better subjective evaluation of performance by subjects (Q5,Q6). Interestingly, logarithmic mapping was more intuitive than linear one (Q1 - Mann-Whitney U test $p=0.04)$ and more effective in reducing the visual burden (Q8 - Mann-Whitney $\mathrm{U}$ test $p=0.047$ ) needed to discriminate different spheres. Furthermore, although the differences are not statistically significant, logarithmic mapping produced a more comfortable and pleasant sensation (Q2,Q3) than the linear one, and to enable an easier discrimination of the position of the wheel on the forearm (Q11,Q12).

\section{Cylinder-size discrimination task}

Results of the cylinder-size discrimination task of 16 subjects, who were tested using four stimuli, are shown in Figure 9. Figure 14 shows the average accuracy for each subject: it can be seen that the results are comparable to those that were found in the sphere-size discrimination task, with an average accuracy of $75.6 \%$ under the haptic feedback condition and an average accuracy of $34.1 \%$ for the no haptic feedback condition. Statistical analysis for this experiment was done using the non-parametric Mann-Whitney-Wilcoxon test for the average performances of the two groups, with a significant difference being found $(p<0.001)$. Figure 13 shows the pairwise results for this test, for which it can be noticed that the haptic feedback condition outperforms the no haptic feedback condition for each pair. These outcomes confirm that the HapPro can be successfully used to convey proprioceptive information, even in an active grasp configuration where the user is reaching to grasp an object.

\section{E. Discussion}

Experimental outcomes show that the HapPro can be successfully used to convey proprioceptive feedback both when an object is placed in the prosthetic hand resting on a table and when users are actively moving it to grasp objects in front of them. In particular, an accuracy of around $75 \%$ was observed in both conditions as opposed to the $33 \%$ observed in the no haptic feedback condition. In previous contributions to this field (e.g., [16], [11], [14], [18]) the effectiveness of proprioceptive feedback was evaluated in virtual reality environments. In this work the setup was tested with a real prosthetic hand both under passive (users closing the hand on an object that was placed in it) and active (users moving the hand on their own to reach and grasp an object) grasp conditions. Thus, the setup seems to be promising for use with amputees. For this reason in the next section we evaluate the setup in a preliminary amputee experiment. A limitation that needs to be tackled is however the relatively large size of the device, not yet suitable for the integration in a prosthetic socket.

\section{Preliminary Experiments With One Amputee SUBJECT}

\section{A. Experiment description}

To further prove the effectiveness of HapPro, we tested the device with one amputee participant (Sub1, Female, 22), who had a right arm transradial amputation. Sub1 was asked to perform the sphere-size recognition task described previously: a short video describing the experiment is shown in [39]. As shown in Fig. 15, the HapPro was placed on the residual limb, without the socket, while the SoftHand Pro (SHP) was remotely controlled through two EMG sensors. Leveraging upon the results we obtained during the experimental session with able bodied participants, we chose logarithmic mapping between the SHP and the HapPro. After a brief training (10 minutes), as described in Section $\mathrm{V}$, the experimenter sequentially presented two randomly chosen wood spheres to the blindfolded subject, who was asked to control the artificial hand to grasp the item and to identify which one was the larger one or if the two specimens had the same dimensions. Results were very positive: Sub1 exhibited a total accuracy of $90 \%$, while the pairwise accuracy is shown in Fig. 16. Considering the comparisons between spheres with very dissimilar dimensions (highlighted with a thick border in the figure), accuracy was $100 \%$, while with spheres with very similar dimensions the accuracy was $83 \%$.

The HapPro was then moved proximally along the arm and positioned above the elbow, while the subject wore the hand prosthesis (Fig. 17). The subject was then given approximately ten minutes to use the SHP while receiving feedback from the HapPro, in order to give her the possibility of making a qualitative comparison between the different positions for the HapPro. Since the subject described the overall experience with this configuration as "slightly unpleasant", the experimenter stopped the experiment without continuing with further quantitative evaluations.

\section{B. Discussion and input for future work}

Sub1 described the difference between the two conditions. In the first case, when the HapPro was positioned on the forearm, the sensation was perceived as more natural. Indeed, in this case the stimuli elicited by the movement of the wheel, which is the result of muscular activity in the residual limb 


\section{SPHERES DISCRIMINATION TASK - PAIRWISE ACCURACY}
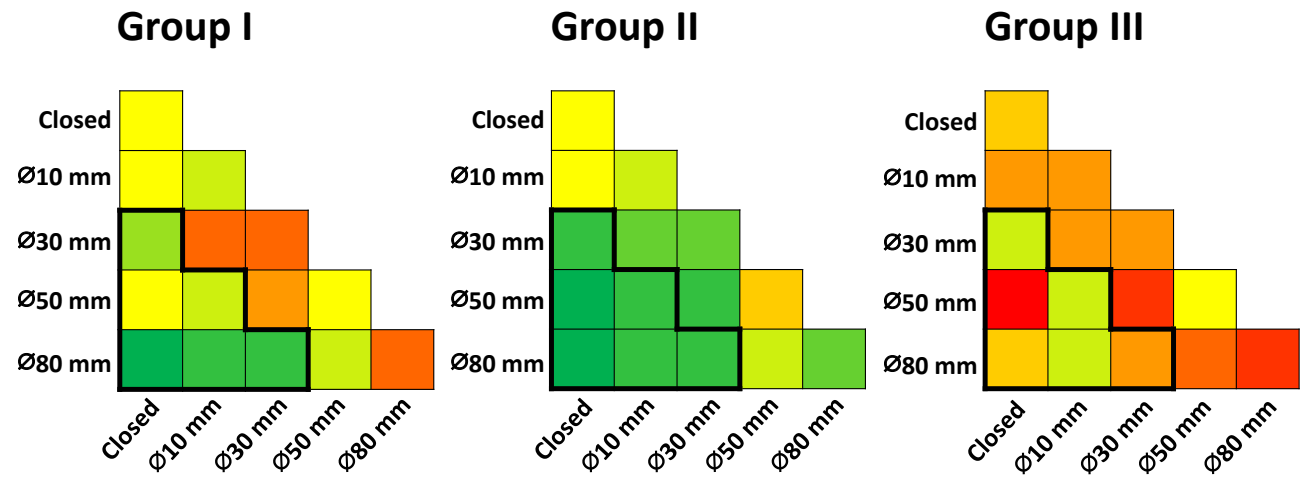

Accuracy (\%)

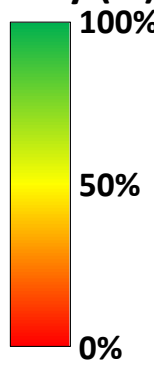

Figure 12: Pairwise accuracy obtained on average by the members of Group I (HapPro ON with linear mapping), Group II (HapPro ON with logarithmic mapping) and Group III (HapPro OFF) during the sphere-size discrimination task. Each square represents the average accuracy obtained by the corresponding group of subjects for that particular pair of spheres. For instance: all of the subjects of Group I and II were able to correctly identified the $80 \mathrm{~mm}$ sphere from the closed hand (dark green square) all of the times. The same square for Group III is orange, corresponding to a value of around $40 \%$, which means that, of all of the trials performed by the subjects of Group III, only $40 \%$ correspond to successful identifications.

\begin{tabular}{|l||c|c|c|}
\hline & Questions & GR I & GR II \\
\hline \hline Q1 & It has been easy to use the SoftHand together with the HapPro. & $5.0 \pm 0.7$ & $6.2 \pm 0.4$ \\
\hline Q2 & I was feeling uncomfortable while using the SoftHand together with the HapPro. & $3.6 \pm 2.3$ & $2.2 \pm 0.8$ \\
\hline Q3 & The sensation provided by the HapPro on the forearm felt pleasant. & $3.8 \pm 1.5$ & $4.4 \pm 1.5$ \\
\hline Q4 & The sensation provided by the HapPro on the forearm felt unpleasant. & $3.4 \pm 2.5$ & $2.8 \pm 0.8$ \\
\hline Q5 & I had the feeling of performing better while receiving position feedback by the HapPro. & $5.2 \pm 1.5$ & $6.2 \pm 0.4$ \\
\hline Q6 & I had the feeling of performing better when I was not receiving any feedback by the HapPro. & $2.8 \pm 1.5$ & $2.2 \pm 1.1$ \\
\hline Q7 & It has been easy to discriminate the spheres. & $4.0 \pm 1.6$ & $4.2 \pm 1.3$ \\
\hline Q8 & Discriminating the spheres without looking at them was very difficult. & $6.2 \pm 1.1$ & $3.8 \pm 1.1$ \\
\hline Q9 & When I was using the HapPro, I was able to tell how open the SoftHand was without looking at it. & $5.8 \pm 0.8$ & $5.4 \pm 0.5$ \\
\hline Q10 & When I was using the HapPro, I had no clue about the opening of the SoftHand. & $1.8 \pm 1.1$ & $1.6 \pm 0.5$ \\
\hline Q11 & It was easy to feel the position of the wheel. & $4.6 \pm 1.5$ & $5.2 \pm 0.8$ \\
\hline Q12 & It was not easy to feel the position of the wheel. & $3.4 \pm 1.9$ & $3.0 \pm 1.4$ \\
\hline Q13 & During the discrimination task, the SoftHand, the HapPro and the spheres were out of my visual field. & $6.4 \pm 0.5$ & $6.6 \pm 0.5$ \\
\hline Q14 & During the discrimination task, I was able to see the spheres or the devices. & $1.2 \pm 0.4$ & $1.2 \pm 0.4$ \\
\hline Q15 & During the discrimination task, I was well isolated from external noises. & $6.6 \pm 0.5$ & $6.6 \pm 0.5$ \\
\hline Q16 & During the discrimination task, I was able to hear the sounds made by the motors of the devices. & $1.2 \pm 0.4$ & $1.4 \pm 0.5$ \\
\hline Q17 & I would have been happy to continue the experiment for longer. & $4.6 \pm 1.9$ & $5.2 \pm 1.9$ \\
\hline Q18 & At the end of the experiment I felt tired. & $5.4 \pm 0.9$ & $4.0 \pm 1.9$ \\
\hline
\end{tabular}

Table I: These statements, presented in random order, were rated by the subjects using a 7-point Likert scale (1: Strongly disagree, 7: Strongly agree). Means and standard deviations for each group are reported.

and the consequent movement of the SHP, were perceived in the same area from which the muscular activity originates. The fact that the efferent and afferent signals to and from the SHP were localized in the same area could help to make the association between action and perception inputs more intuitive, thus contributing to better discriminate the level of closure of the SHP without visual cues and with much less cognitive effort. Furthermore, since this location is closer to the phantom hand and to the site of tactile stimuli delivery in intact persons, this could make somatotopic matching stronger [13].

In the second case, i.e. when the HapPro was positioned above the elbow, the association between the movements of the SHP and the movements of the HapPro was felt as counter- intuitive. Sub1 was still able to roughly understand the level of closure of the SHP from the sensations provided by the HapPro, but it required more cognitive effort due to the difficulty in associating the stimulation of the skin above the elbow to the movement of the prosthesis.

In summary, we have shown that combining the ease of control and robustness of the SoftHand Pro and the usefulness of proprioceptive feedback provided by the HapPro, size recognition tasks can be easily performed by prosthesis users. The work done with the amputee also showed how important it is to have a device integrated in the socket. In transradial amputees, a situation where there is not enough space to place both a socket and a haptic device on the forearm is not uncommon; for this reason, to profitably employ in real world 
CYLINDERS DISCRIMINATION TASK - PAIRWISE ACCURACY
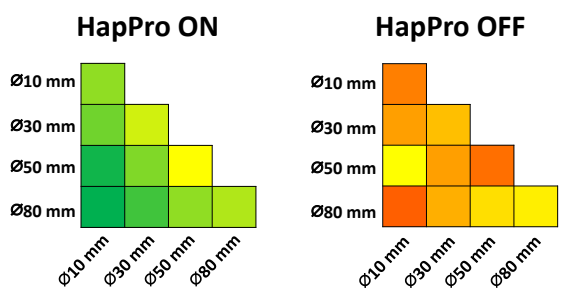

Accuracy (\%)

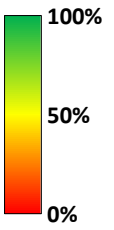

Figure 13: Pairwise accuracy obtained on average by the subjects of the two conditions during the cylinder-size discrimination task. In the condition HapPro ON the HapPro was used with the logarithmic mapping, while in the condition HapPro OFF, the HapPro was not giving any feedback to the subjects.

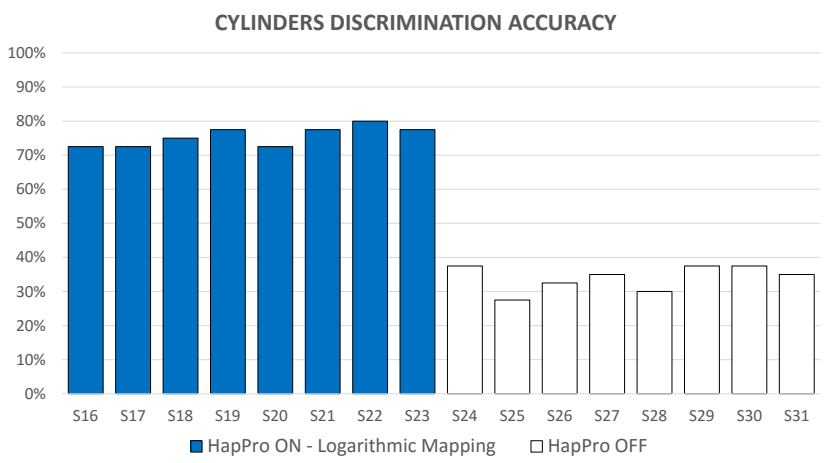

Figure 14: Discrimination accuracy obtained by the members of the two groups during the cylinder-size discrimination task.

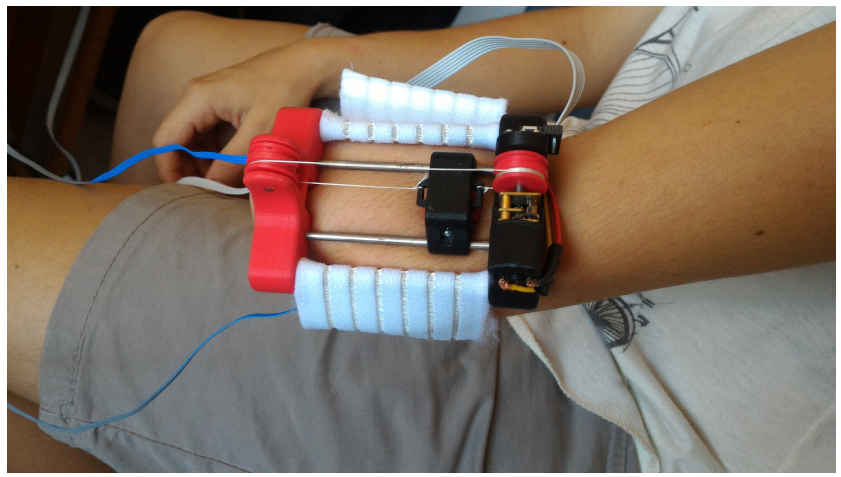

Figure 15: In the configuration shown in the picture, the HapPro is positioned in the distal part of the residual limb along with the EMG sensors. The SHP (not shown in this figure) is placed on a table in front of the subject.

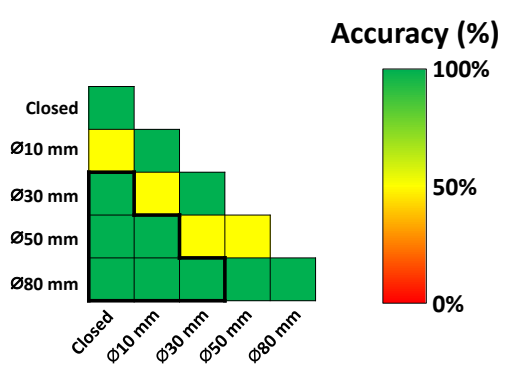

Figure 16: Pairwise accuracy obtained on average by Sub1 during the sphere-size discrimination task. For each pair of spheres, the accuracy was calculated averaging the results of 2 discrimination trials.

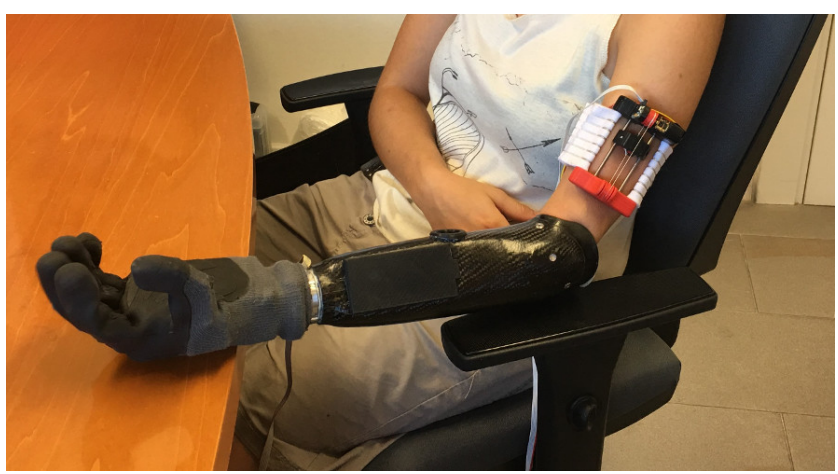

Figure 17: In the configuration shown in the picture, the HapPro is positioned above the elbow, in order to leave the distal portion of the residual limb free so that the socket can be placed on it.

application, a device such as the one we proposed, having a design that is compact and lightweight enough to be integrated is a mandatory step.

\section{CONCLUSION}

We presented the HapPro, a wearable haptic device used to convey proprioceptive information on the aperture of a synergy-based prosthetic hand. This is done through a moving wheel on the user's forearm, mapping the encoder-measured hand aperture to the position of the wheel using logarithmic and linear relationships. Psychometric results showed that the anterior side of the forearm was more sensitive than the posterior side, we chose this site for perceptual experiments, where the effectiveness of HapPro was tested in conjunction with a myoelectric version of the Pisa/IIT SoftHand in size discrimination tasks.

Experimental outcomes reveal that HapPro provided a good level of accuracy for size discrimination, as measured in two different grasping tasks, as the results advance the work by others in the virtual world. Accuracy was first tested with the prosthetic hand resting on a table. Accuracy remained high when the prosthetic hand was moved to reach and grasp for objects. Survey results also showed it was intuitive and effective, with logarithmic mapping exhibiting the best results. We also performed a proof-of-concept experiment with an 
amputee subject, which, to the best of our knowledge, was never done before for a proprioceptive feedback device.

In conclusion, the Hap-Pro can be a viable solution to provide proprioceptive feedback on artificial hand opening when used with an under-actuated synergy-inspired robotic manipulator. Future work will focus on further testing this system with amputees as well as for other assistive systems and robotic tele-operation. Furthermore, the experiment with the amputee showed that it would be desirable for the device to be smaller and lighter, to integrate the haptic systems into the prosthetic socket. A new design will address these issues.

\section{ACKNOWLEDGMENT}

This research has received funding from the European Union's Horizon 2020 Research and Innovation Programme under Grant Agreement No.688857 (SoftPro). The content of this publication is the sole responsibility of the authors. The European Commission or its services cannot be held responsible for any use that may be made of the information it contains.

\section{REFERENCES}

[1] A. Chadwell et al., "The reality of myoelectric prostheses: Understanding what makes these devices difficult for some users to control," Frontiers in Neurorobotics, vol. 10, no. 5, pp. 1-21, 2016.

[2] D. Datta, K. Selvarajah, and N. Davey, "Functional outcome of patients with proximal upper limb deficiency -acquired and congenital." Clinical Rehabilitation, vol. 18, no. 2, pp. 172-177, 2004.

[3] E. A. Biddiss and T. T. Chau, "Upper limb prosthesis use and abandonment: a survey of the last 25 years," Prosthetics and Orthotics International, vol. 31, no. 3, pp. 236-257, 2007.

[4] C. Pylatiuk, S. Schulz, and L. Döderlein, "Results of an internet survey of myoelectric prosthetic hand users," Prosthetics and Orthotics International, vol. 31, no. 4, pp. 362-370, 2007.

[5] E. Biddiss, D. Beaton, and T. Chau, "Consumer design priorities for upper limb prosthetics," Disability and Rehabilitation: Assistive Technology, vol. 2, no. 6, pp. 346-357, 2007.

[6] S. Lewis et al., "User demands for sensory feedback in upper extremity prostheses," in Proceedings of the IEEE International Symposium on Medical Measurements and Applications (MeMeA), 2012, pp. 1-4.

[7] P. D. Marasco et al., "Robotic touch shifts perception of embodiment to a prosthesis in targeted reinnervation amputees," Brain, vol. 134, no. 3, pp. 747-758, 2011.

[8] A. Ninu et al., "Closed-loop control of grasping with a myoelectric hand prosthesis: Which are the relevant feedback variables for force control?" IEEE Transactions on Neural Systems and Rehabilitation Engineering, vol. 22, no. 5, pp. 1041-1052, 2014.

[9] H. J. Witteveen et al., "Vibro-and electrotactile user feedback on hand opening for myoelectric forearm prostheses," IEEE Transactions on Biomedical Engineering, vol. 59, no. 8, pp. 2219-2226, 2012.

[10] A. Ajoudani et al., "Exploring teleimpedance and tactile feedback for intuitive control of the Pisa/IIT SoftHand," IEEE Transactions on Haptics, vol. 7, no. 2, pp. 203-215, 2014.

[11] K. Bark et al., "Rotational skin stretch feedback: A wearable haptic display for motion," IEEE Transactions on Haptics, vol. 3, no. 3, pp. 166-176, 2010.

[12] S. Casini et al., "Design and realization of the cuff-clenching upperlimb force feedback wearable device for distributed mechano-tactile stimulation of normal and tangential skin forces," in Proceedings of the IEEE International Conference on Intelligent Robots and Systems (IROS), 2015, pp. 1186-1193.

[13] K. Kim and J. E. Colgate, "Haptic feedback enhances grip force control of semg-controlled prosthetic hands in targeted reinnervation amputees," IEEE Transactions on Neural Systems and Rehabilitation Engineering, vol. 20, no. 6, pp. 798-805, 2012.

[14] A. Blank, A. M. Okamura, and K. J. Kuchenbecker, "Identifying the role of proprioception in upper-limb prosthesis control: Studies on targeted motion," ACM Transactions on Applied Perception, vol. 7, no. 3, p. 15, 2010.

[15] D. J. Atkins, D. C. Heard, and W. H. Donovan, "Epidemiologic overview of individuals with upper-limb loss and their reported research priorities." Journal of Prosthetics and Orthotics, vol. 8, no. 1, pp. 2-11, 1996.
[16] A. Akhtar et al., "Passive mechanical skin stretch for multiple degreeof-freedom proprioception in a hand prosthesis," in Proceedings of the Springer International Conference on Human Haptic Sensing and Touch Enabled Computer Applications, 2014, pp. 120-128.

[17] M. G. Catalano et al., "Adaptive synergies for the design and control of the Pisa/IIT SoftHand," International Journal of Robotics Research, vol. 33, no. 5, pp. 768-782, 2014.

[18] A. Cheng et al., "Conveying the configuration of a virtual human hand using vibrotactile feedback," in Proceedings of the IEEE Haptics Symposium. IEEE, 2012, pp. 155-162.

[19] M. Santello, M. Flanders, and J. F. Soechting, "Postural hand synergies for tool use," Journal of Neuroscience, vol. 18, no. 23, pp. 10105 10115,1998

[20] D. I. McCloskey, "Kinesthetic sensibility." Physiological Reviews, vol. 58 , no. 4 , pp. $763-820,1978$.

[21] D. F. Collins et al., "Cutaneous receptors contribute to kinesthesia at the index finger, elbow, and knee," Journal of Neurophysiology, vol. 94, no. 3, pp. 1699-1706, 2005.

[22] T. Pistohl et al., "Artificial proprioceptive feedback for myoelectric control," IEEE Transactions on Neural Systems and Rehabilitation Engineering, vol. 23, no. 3, pp. 498-507, 2015.

[23] R. Mann and S. Reimers, "Kinesthetic sensing for the emg controlled "Boston Arm"," IEEE Transactions on Man-Machine Systems, vol. 11, no. 1, pp. 110-115, 1970

[24] R. Christiansen et al., "Vibrotactile feedback of pose error enhances myoelectric control of a prosthetic hand," in Proceedings of the IEEE World Haptics Conference (WHC). IEEE, 2013, pp. 531-536.

[25] J. S. Schofield et al., "Applications of sensory feedback in motorized upper extremity prosthesis: a review," Expert Review of Medical Devices, vol. 11 , no. 5, pp. 499-511, 2014.

[26] C. J. Hasson and J. Manczurowsky, "Effects of kinematic vibrotactile feedback on learning to control a virtual prosthetic arm," Journal of Neuroengineering and Rehabilitation, vol. 12, no. 31, pp. 1-16, 2015.

[27] K. A. Kaczmarek et al., "Electrotactile and vibrotactile displays for sensory substitution systems," IEEE Transactions on Biomedical Engineering, vol. 38, no. 1, pp. 1-16, 1991.

[28] J. Wheeler et al., "Investigation of rotational skin stretch for proprioceptive feedback with application to myoelectric systems," IEEE Transactions on Neural Systems and Rehabilitation Engineering, vol. 18 , no. 1 , pp. 58-66, 2010.

[29] K. Bark et al., "Comparison of skin stretch and vibrotactile stimulation for feedback of proprioceptive information," in Proceedings of the IEEE Symposium on Haptic Interfaces for Virtual Environment and Teleoperator Systems, 2008, pp. 71-78.

[30] W. R. Provancher et al., "Contact location display for haptic perception of curvature and object motion," International Journal of Robotics Research, vol. 24, no. 9, pp. 691-702, 2005.

[31] S. B. Godfrey et al., "The SoftHand Pro: Translation from robotic hand to prosthetic prototype," in Converging Clinical and Engineering Research on Neurorehabilitation II. Springer, 2017, pp. 469-473.

[32] P. H. Thakur, A. J. Bastian, and S. S. Hsiao, "Multidigit movement synergies of the human hand in an unconstrained haptic exploration task," The Journal of Neuroscience, vol. 28, no. 6, pp. 1271-1281, 2008.

[33] A. Bicchi, M. Gabiccini, and M. Santello, "Modelling natural and artificial hands with synergies," Philosophical Transactions of the Royal Society B, vol. 366, no. 1581, pp. 3153-3161, 2011.

[34] C. Della Santina et al., "The quest for natural machine motion: An open platform to fast-prototyping articulated soft robots," IEEE Robotics \& Automation Magazine, vol. 24, no. 1, pp. 48-56, 2017.

[35] G. A. Gescheider, Psychophysics: the fundamentals. Psychology Press, 2013.

[36] A. Moscatelli et al., "The change in fingertip contact area as a novel proprioceptive cue," Current Biology, vol. 26, no. 9, pp. 1159-1163, 2016.

[37] M. Bianchi and A. Serio, "Design and characterization of a fabric-based softness display," IEEE Transactions on Haptics, vol. 8, no. 2, pp. 152163,2015

[38] A. Moscatelli, M. Mezzetti, and F. Lacquaniti, "Modeling psychophysical data at the population-level: the generalized linear mixed model," Journal of Vision, vol. 12, no. 11, pp. 26-26, 2012.

[39] (2017) A video of the experiments with the amputee subject. [Online]. Available: https://www.dropbox.com/s/u317msffzrnpcgj/HapPro-HD\% 20720p\%20copy.mov?dl=0 